\title{
Vacuna anti-pertussis para uso en adolescentes y adultos
}

\author{
Cecilia Perret $P$.
}

\section{Pertussis vaccine for adolescents and adults}

After the introduction of the pertussis vaccine the number of cases decreased substantially, albeit, the disease remains present affecting mostly young infants in whom it causes severe complications, adolescents and adults. Adults are the main reservoir of Bordetella pertussis and serve as vehicle of transmission reservoir to infants. The new pertussis vaccines, manufactured for adolescents and adults, have good immunogenicity, safety and efficacy (92\%) profile. The introduction of this vaccine into a universal vaccination program for adolescents and adults, would decrease the incidence of the disease in this population and more importantly, transmission of the microorganism to infants.

Key words: pertussis vaccine, pertussis, adolescents, adults, immunogenicity, efficacy, review.

Palabras claves: vacuna anti-pertussis, coqueluche, adolescentes y adultos, inmunogenicidad, eficacia, revisión.

\section{Introducción}

$\mathrm{L}$ uego de la introducción de la vacuna antipertussis en la población infantil, alrededor de los años 60 se observó una marcada disminución de las tasas de morbilidad y mortalidad por coqueluche (= tos convulsiva). Sin embargo, luego de haberse alcanzado una situación basal, desde mediados de los 90 se comenzó a observar un nuevo aumento en el número de casos de esta afección. Estos nuevos casos correspondían principalmente a lactantes bajo 1 año de edad, adolescentes y adultos ${ }^{1-3}$. En Chile, las tasas de casos de tos convulsiva entre los años 1996 y 2000 aumentaron a 25/100.000 hab, casi comparables a la década de los 80 . Actualmente han descendido a valores de 7/100.000 hab. Explicaciones para este fenómeno son muchas pero entre las más aceptadas se encuentran: la disponibilidad de mejores métodos de diagnóstico, mayor reconocimiento por los médicos de la tos convulsiva en los adolescentes y adultos, mejoría en las notificaciones. Estos factores han contribuido a la existencia de una "pseudoepidemia" por aumento en el diagnóstico y notificaciones. No obstante, un incremento real de los casos ha ocurrido como resultado de una pérdida de la inmunidad luego de 5 a 10 años de haberse efectuado la vacunación primaria (2 - 4 - 6 - 18 meses) y el refuerzo en el pre-escolar, con la vacuna anti-pertussis elaborada con bordetela inactivada (vacuna de "células completas") y la consiguiente acumulación de susceptibles en edades mayores ${ }^{2-4}$.
Los adolescentes y adultos se han transformado en una fuente de transmisión entre sus pares y hacia los lactantes menores ${ }^{5-7}$. Estudios nacionales, así como los efectuados en otros países, avalan esta afirmación. En un estudio llevado a cabo en Santiago, sobre 10 grupos familiares de lactantes bajo 6 meses de edad, que fueran hospitalizados por coqueluche, se evidenció que al menos una persona en cada grupo familiar tenía Bordetella pertussis en su secreción nasofaríngea. Se identificó el caso primario en 8 de 10 grupos familiares, con una edad promedio de 25,7 años ${ }^{8}$.

La vacunación al grupo de adolescentes y adultos podría ser una buena estrategia para disminuir las tasas de morbilidad por coqueluche en estos grupos pero también podría contribuir a la reducción de casos en lactantes menores al disminuir la fuente de contagio que representan adolescentes y adultos.

El desarrollo de vacunas acelulares, que contienen antígenos purificados de $B$. pertussis, y cuya reactogenicidad es significativamente menor que las vacunas de células completas, abrió un camino para el diseño de productos aplicables en la población de mayor edad, en quienes se encontraba contraindicado el uso de vacunas celulares por el riesgo de eventos adversos graves.

\section{Vacunas anti-pertussis en adolescentes}

En el último año se han licenciado dos vacunas acelulares combinadas con toxoide tetánico y diftérico, especialmente formuladas para adolescentes y adultos. Estas vacunas tienen dosis reducidas de todos
Pontificia Universidad Católica de Chile Facultad de Medicina Departamento de Pediatría. Unidad de Infectología.

Recibido: 23 febrero 2006 Aceptado: 4 abril 2006

Correspondencia a: Cecilia Perret Pérez cperret@med.puc.cl 
sus componentes (dTpa), pudiendo entonces ser utilizadas a partir de los 7 años.

Se han realizados varios estudios de inmunogenicidad y seguridad sobre los productos actualmente licenciados: Boostrix ${ }^{\circledR}$ de Glaxo SmithKlein y Daptacel ${ }^{\circledR}$ de Sanofi Pasteur. La primera vacuna está licenciada en Chile pero al momento de escribir este artículo aún no disponible en el mercado, la segunda está en trámite de registro en el ISP. A continuación se presentan los antecedentes de estas dos vacunas.

\section{Daptacel ${ }^{\circledR}$ de Sanofi Pasteur}

Se realizó un estudio de inmunogenicidad y seguridad controlado, contra una vacuna que contenía toxoides diftérico y tetánico (dT), randomizado y doble ciego, en una población de 11 a 64 años de edad ${ }^{9}$. La vacuna dTpa utilizada contiene toxoide diftérico: 2 límites de floculación (Lf), toxoide tetánico: 5 Lf; 5 antígenos de $B$. pertussis: 2,5 $\mu \mathrm{g}$ de pertusinógeno (TP), $5 \mu \mathrm{g}$ de hemaglutinina filamentosa (HAF), $3 \mu \mathrm{g}$ pertactina $(\mathrm{PRN})$ y $5 \mu \mathrm{g}$ de fimbrias tipo 2 y 3 . Al grupo en estudio se le administró una dosis de refuerzo de dTpa y se comparó con un grupo control que recibió un refuerzo de dT. Los grupos fueron estratificados por edad para asegurar tener el mismo número en la población de estudio y control. Se analizó en ambos grupos la presencia de eventos adversos y la respuesta inmune midiendo los títulos de anticuerpos para cada uno de los antígenos. Se obtuvieron muestras de

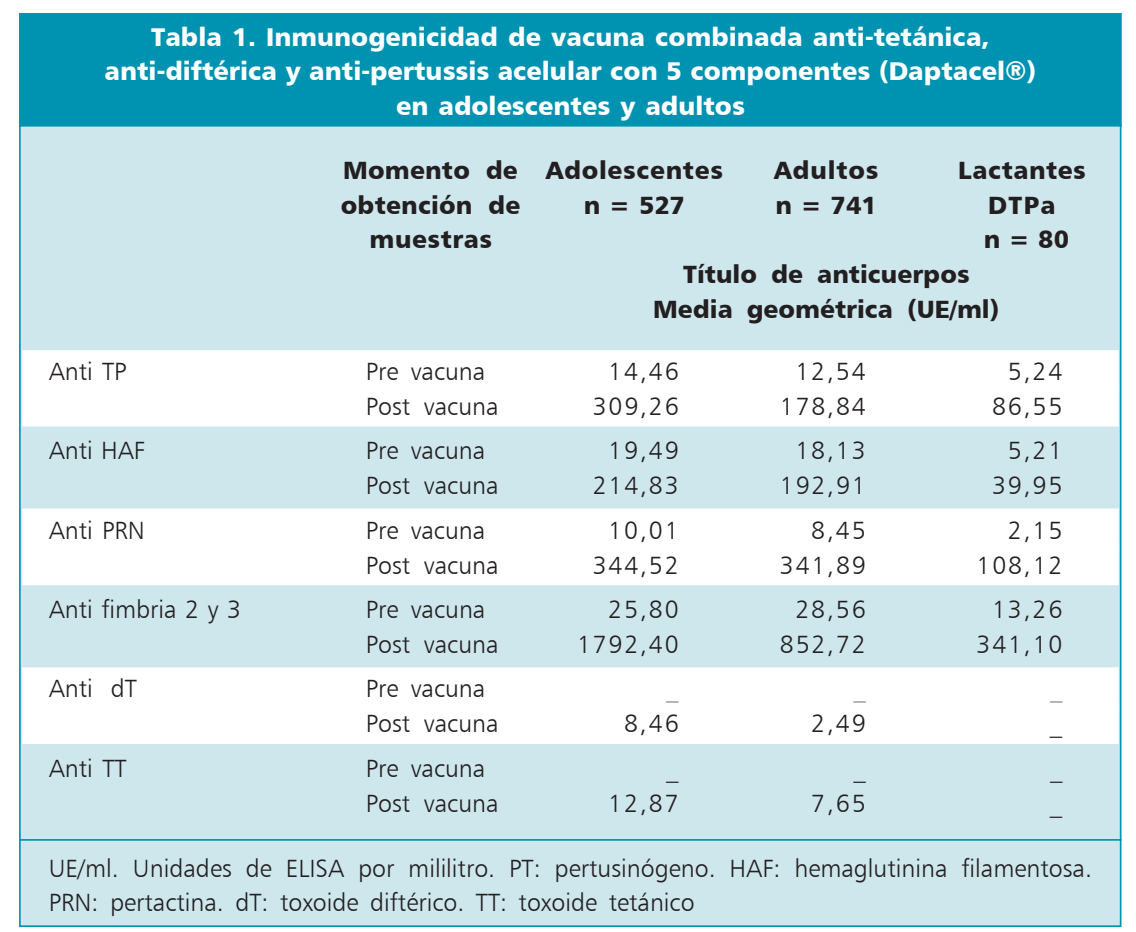

sangre antes de la vacunación y a los 28 y 42 días post vacuna. Los títulos de anticuerpos anti-pertussis se compararon con los generados luego de la vacunación primaria de tres dosis en una población de 80 lactantes. Resultados: Participaron finalmente en el estudio 2.296 individuos. La seroprotección generada contra difteria y tétanos $(>0,1 \mathrm{IU} / \mathrm{ml})$ fue comparable entre ambos grupos, evaluadas al mes después de la vacunación. Los títulos de anticuerpos para todos los antígenos de pertussis fueron significativamente más altos en la población adolescentes adultos que en los lactantes. Se obtuvo respuesta tipo booster, en más de $80 \%$ de la población vacunada, para todos los antígenos del componente anti-pertussis y fue similar en la población de adolescentes y de adultos (Tabla 1). Las reacciones locales como dolor, aumento de volumen y eritema fueron similares en ambos grupos. No hubo diferencias significativas en las manifestaciones sistémicas como fiebre, cefalea ni malestar general entre los dos grupos. En conclusión, esta vacuna genera una adecuada respuesta inmune, tanto para los antígenos dT como de $B$. pertussis, en la población de adolescentes $\mathrm{y}$ adultos. Es una vacuna segura y su reactogenicidad es similar a la observada con la vacuna dT. El grupo investigador concluyó que su aplicación en E.U.A. podría reducir la incidencia y el reservorio de la enfermedad en la población teniendo como resultado una disminución de la transmisión desde adolescentes y adultos a los lactantes.

\section{Boostrix ${ }^{\circledR}$ de Glaxo SmithKlein}

Esta vacuna ha sido evaluada con ensayos controlados contra dT, randomizados, doble ciego, al igual que Daptacel®. Se analizan a continuación antecedentes de un estudio de inmunogenicidad y seguridad realizado en población chilena ${ }^{10}$. La vacuna dTpa utilizada combina 2 UI de toxoide diftérico, 20 UI de toxoide tetánico, y 3 antígenos de $B$. pertussis: $8 \mu \mathrm{g}$ de TP, $8 \mu \mathrm{g}$ de HAF y $2,5 \mu \mathrm{g}$ PRN. Las cantidades son bastante menores que las utilizadas en la vacuna pediátrica DTPa. Mediante un estudio abierto se compararon dos grupos de individuos: de 10 a 11 años y 18 años o más. A la población se le aplicó una dosis de refuerzo de la vacuna en estudio y se siguió durante 14 días para vigilar la aparición de eventos adversos. Se obtuvo una muestra de sangre previa a la vacunación y 30 días posteriores a ella para la determinación de títulos de anticuerpos específicos contra los antígenos contenidos en la vacuna. Resultados: Participaron en el estudio 120 individuos, 60 niños de 10 a 11 años y 60 adultos con 18 años o más. En la población adulta 50\% de los individuos era seropositivo para PT, 98,3\% para HAF y $85 \%$ para PRN, previo a la vacunación. El 100\% de los individuos, tanto adultos como niños, fueron 
seropositivos al mes post vacunación. Tres individuos adultos fueron considerados no respondedores porque, siendo seropositivos, no lograron duplicar sus títulos basales. Los títulos aumentaron significativamente para los 3 antígenos de $B$. pertussis en la población estudiada con incrementos de al menos 14 veces el valor basal. En este estudio destacó además que sólo 58,3 y $27,1 \%$ de la población adulta era seropositiva para los anticuerpos anti-diftéricos y antitetánicos, respectivamente. Esta situación se revirtió post vacunación alcanzando una seropositividad con títulos protectores en $96,7 \%$ de la población contra ambos toxoides. No ocurrió reacción inmediata alguna a la vacuna ni se registraron eventos adversos graves. Durante el período de seguimiento $91,7 \%$ de los individuos presentó algún síntoma local, siendo dolor el más frecuente; $51,7 \%$ de los niños y $75 \%$ de los adultos presentaron algún síntoma sistémico. Estos resultados son comparables a los obtenidos en otros estudios controlados con dT. Conclusiones: Esta vacuna mostró una adecuada respuesta inmune a todos los componentes contenidos en ella y presentó un adecuado perfil de seguridad como para ser aplicada en la población de adolescentes y adultos. Un porcentaje elevado de la población adulta chilena no posee anticuerpos anti-tetánicos y en menor proporción anticuerpos anti-diftéricos. Esto respalda la recomendación de refuerzo, al menos de dT, cada 10 años. El uso de esta vacuna sería un avance significativo en el camino del control de la circulación de B. pertussis protegiendo así a los lactantes pequeños contra el riesgo de contagio.

\section{Eficacia de vacuna anti-pertussis en adolescentes $y$ adultos}

Un solo estudio ha sido publicado hasta la fecha en que se evalúa la eficacia de las vacunas de adultos. Este fue realizado utilizando la formulación de GlaxoSmithKlein en personas entre 15 y 65 años de edad $^{11}$. Los individuos fueron seguidos por una mediana de 22 meses. Los casos de coqueluche fueron definidos como tos mantenida durante 5 o más días, y confirmados por cultivo, RPC o serología (ascenso de títulos entre muestras de fase aguda y convalecien- tes). Con esta definición se estimó una incidencia de coqueluche en este grupo etario de 370 casos por 100.000 personas-año. La eficacia global de la vacuna fue de $92 \%$. La duración de la protección y la prevención de casos secundarios no fueron evaluadas en este estudio.

\section{Conclusiones}

La coqueluche es una de las enfermedades inmunoprevenibles con un deficiente control epidemiológico. Esto se debe a la pérdida de la inmunidad, tanto en las personas vacunadas durante la infancia, como en aquellas que desarrollan la enfermedad natural. Los adolescentes y adultos sirven de reservorio de la infección para los lactantes quienes presentan una mayor morbimortalidad. Probablemente el control de esta enfermedad requiera de la inmunización rutinaria en adolescentes y adultos para reducir la carga y transmisión de B. pertussis. La disponibilidad actual de vacunas que pueden ser aplicadas en la población de adolescentes y adultos, que han demostrado una adecuada inmunogenicidad, perfil de seguridad así como una buena eficacia, hacen posible pensar en programas destinados al control de la coqueluche.

\section{Resumen}

A pesar de la vacunación para prevenirla, la tos convulsiva es una enfermedad aún presente, afectando principalmente a la población de lactantes pequeños con alta morbi-mortalidad, pero también a los adolescentes y adultos que han perdido el efecto de la vacunación inicial; este segundo grupo se ha transformado en el principal reservorio y fuente de transmisión de Bordetella pertussis a lactantes. Las nuevas vacunas anti-pertussis, especialmente formuladas para este grupo etario, tienen un buen perfil de inmunogenicidad y de seguridad y su eficacia alcanza a $92 \%$. La utilización universal de esta vacuna podría contribuir al control de la tos convulsiva en los grupos más susceptibles.

\section{Referencias}

1.- Sánchez I, Repetto G, Saenger A. Epidemiología de la tos ferina en Chile (1950-1990). El adulto, ¿nuevo reservorio de infección? Rev Méd Chile 1994; 122: 339-45.

2.- Bass J W, Wittler R R. Return of epidemic pertussis in the United States. Pediatr Infect
Dis J 1994; 13: 343-5.

3.- De Melker H, Schellenkens J E P, Neppelenbroek S E, Mooi Fr, Rumke H C, Conyn-Van Spaendonck M. Re-emergence of pertussis in the highly vaccinated population of the Netherlands: Observations on surveillance data. Emerg Infect Dis 2000; 6: $348-57$.
4.- Jenkinson D. Duration of effectiveness of pertussis vaccine: evidence from a 10 year community study. Br Med J 1988; 296: 612-4.

5.- Nelson J D. The changing epidemiology of pertussis in young infants. The role of adults as reservoirs of infection. Am J Dis Child 1978; 132: 371-3. 
6.- Crowcroft N S, Booy R, Harrison T, Spicer L, Britto J, Mok Q, et al. Severe and unrecognised: pertussis in UK infants. Arch Dis Child 2003; 88: 802-6

7.- De Schuter I, Malfroot A, Dab I, Hoebrekx N, Muyldermans G, Pierard D, et al. Molecular typing of Bordetella pertussis isolates recovered from Belgian children and their household members. Clin Infect Dis 2003; 36: 1391-6.

8.- Perret C, Viviani T, Peña A M, Abarca K,
Ferrés M. Determinación de la fuente de infección de coqueluche en lactantes menores de 6 meses. XXI Congreso Chileno de Infectología. Viña del Mar 13-16 octubre 2004. resumen CO51, pág 77.

9.- Pichichero M, Rennels M, Edwards K, Blatter M, Marshall G S, Bologa M, et al. Combined tetanus, diphtheria, and 5 component pertussis vaccine for use in adolescents and adults. JAMA 2005; 293 : 3003-11.
10.- Abarca K, Valdivieso F, Potin M, Ibáñez I, Vial P. Inmunogenicidad y seguridad de una vacuna difteria, tétanos, pertussis acelular de contenido antigénico reducido (dTpa) en niños de 10 a 11 años de edad y en adultos. Rev Méd Chile 2002; 130: 502-10.

11.- Ward J, Cherry J, Chang S, Partridge S, Lee H, Treamor J, et al. Efficacy of an acellular pertussis vaccine among adolescents and adults. N Engl J Med 2005; 353: $1555-63$ 